\title{
Rasgos de la estrategia de los cibermedios gallegos en su empeño por promover la experimentación para el cambio de modelo periodístico
}

\author{
Xosé LóPez GARCÍA \\ Universidad de Santiago de Compostela \\ xose.lopez.garcia@usc.es
}

Recibido: 29/12/2009

Aceptado: 11/04/2010

\begin{abstract}
Resumen
Los cibermedios gallegos utilizan muy poco las herramientas de la web social y apenas incorporan servicios de valor añadido. El elemento más empleado es el envío de noticias por correo electrónico, seguido de la posibilidad de imprimir contenidos y la sindicación. También es muy limitada la posibilidad de visualizar la información en PDF y el recurso de hemeroteca, lo que demuestra que la mayoría de los cibermedios gallegos tienen un largo camino por recorrer en el aprovechamiento de las posibilidades del nuevo modelo. Sin embargo, los responsables de los cibermedios afirman que apuestan por la información en la red y que consideran las herramientas de la web social una oportunidad para el cambio de modelo periodístico que ha comenzado en los últimos años de la mano de internet.
\end{abstract}

Palabras clave: Ciberperiodismo, herramientas de la web social, información en internet, cambio periodístico.

\section{Traits of the strategy of Galician online media in his determination for promoting the experimentation for the journalistic model change}

\begin{abstract}
Galician online media hardly ever use social web tools or added value services. The elements more used are the news order by email, the chance of printing contents, the RSS feeds and the possibility of changing the letters size. They are very limited too the chance of visualizing news on PDF and the newspaper library resource, proving that most of Galician online media have even a long way to cover for taking advantage of Internet news possibilities. In spite of actual situation, online media responsibles declare that they bet on information on line and they think about social web like an opportunity to change the journalist model started some years ago thanks to Internet.
\end{abstract}

Key words: CyberJournalism, Social Web Tools, Internet News, Journalistic Change.

\section{Referencia normalizada}

LÓPEZ GARCÍA, Xosé (2011): "Rasgos de la estrategia de los cibermedios gallegos en su empeño por promover la experimentación para el cambio de modelo periodístico". Estudios sobre el mensaje periodístico. Vol. 17, núm. 1, págs.: 81-93. Madrid, Servicio de Publicaciones de la Universidad Complutense.

Sumario: 1. Introducción. Cibermedios en fase de cambio. 2. En busca de usuarios mediante una información creíble. 3. El llamado "Periodismo ciudadano" como experiencia para analizar. 4. La red como espacio de deliberación pública. 5. La interactividad y la participación como motores del periodismo. 6. Algunos rasgos del nuevo escenario. 7. Conclusión. 8. Referencias bibliográficas. 


\section{Introducción}

Los cibermedios que apuestan por el modelo tradicional de periodismo y la participación de los usuarios avanzan lentamente en la incorporación de las herramientas de la web social. Esta es, al menos, la conclusión que se extrae de la investigación realizada en el año 2009 sobre los cibermedios existentes en Galicia ${ }^{1}$, la mayoría promovidos por las empresas editoras de los catorce diarios de información general existentes en la Comunidad. Los resultados de la investigación constatan que, en términos generales, los cibermedios gallegos siguen pautas tradicionales de tratamiento de la información, desaprovechan algunas de las posibilidades que ofrece la red y presentan algunas deficiencias estructurales que lastran los recursos hipertextuales disponibles en la web.

En el estudio de los cibermedios gallegos se constatan tres tendencias generalizadas que indican la existencia de un alto grado de traslación de la información del diario en papel a la red sin aprovechar muchas de las posibilidades que ofrece la web, un comportamiento heterogéneo en la aplicación de los estándares de facilidad de uso de las webs, y la tendencia generalizada a mantener un nivel bajo de actualización de las noticias. Los cibermedios analizadas ofrecen, en general, una información diseñada desde pautas convencionales en el campo formal, con un claro predominio de los textos informativos llevados a portada -el 92 por ciento- y muy bajos porcentajes de textos interpretativos -7 por cien- y opinativos -1 por cien-.

A pesar de las numerosas herramientas existentes para la participación y para la explicación de la información -especialmente, las herramientas popularizadas en la denominada web social-, su uso es limitado por parte de los cibermedios gallegos. También es poco frecuente el empleo de infografías para mostrar las informaciones -sólo la mitad de los cibermedios analizados emplea alguna infografía-, al tiempo que tienen una escasa presencia las galerías de imágenes, vídeos o archivos directos. Es más frecuente, en cambio, la presencia de elementos interactivos -el más habitual es la valoración de noticias, presente en un 38 por cien de los casos-.

La incorporación a las redes sociales llega en los cibermedios estudiados a un 35 por cien y los comentarios de noticias al 27 por cien. La presencia de otros elementos, como encuestas, foros o chats, es mucho más limitada. En todo caso, en las entrevistas en profundidad a los responsables de las entidades promotoras de los cibermedios gallegos ${ }^{2}$ hay constantes referencias a la importancia de las

${ }^{1}$ Los datos que se recogen en este artículo proceden de los resultados de la primera parte de la investigación "Tendencias na converxencia dos medios de comunicación en Galicia 2007-2010” -proyecto PGIDIT07PXIB212149PR, de la dirección general de I+D de la Xunta de Galicia-. En la investigación se han analizado los once cibermedios más importantes, entre los que están las ediciones digitales de La Voz de Galicia, Faro de Vigo, El Correo Gallego, El Progreso, La Región, Xornal de Galicia y A Nosa Terra.

${ }^{2}$ Las opiniones que se recogen en este artículo corresponden a los resultados de las primeras entrevistas en profundidad con los responsables de los cibermedios gallegos o de las empresas promotoras, así como de las opiniones del grupo de redactores de cibermedios gallegos consultados en las investigaciones que ha realizado el grupo Novos Medios en el año 2009. 
herramientas de la web social y a los proyectos existentes para su incorporación en el futuro inmediato.

\section{Qué, por qué y cómo}

Los cibermedios gallegos consideran básico ofrecer información bien elaborada, con un tratamiento convencional, y dando voz a los usuarios, pero sin entrar en el terreno del conocido como "Periodismo ciudadano", cuya aparición coincidió en el tiempo con una crisis general del consumo de la información en los soportes tradicionales y con momentos críticos para el periodismo ${ }^{3}$ convencional. En la actualidad, a veces los medios de comunicación tradicionales son percibidos por los públicos como demasiado distantes. Y, al mismo tiempo, con la llegada de las tecnologías actuales, las noticias se han convertido en una materia prima con la que es posible elaborar productos de mayor complejidad, con un valor añadido, gracias a la convergencia entre medios y tecnología.

Los datos de lectura y consumo de la industria periodística son contundentes y se posicionan a favor de cambios": es necesaria una revisión y ésta parece que ya está en marcha. Los medios de comunicación nacieron antes como servicio público que como negocio: la prensa daba a sus lectores la información que el público debía de conocer por su interés intrínseco y pragmático. Precisamente esa vocación originaria de servicio público parece ser una de las vías a las que debe aferrarse el periodismo tradicional para salir de la crisis. Y para ello trata de abrir ventanas a la participación para que la configuración de la agenda se nutra más de las aportaciones de los ciudadanos.

Estas iniciativas de interacción, que aplican los cibermedios gallegos, aunque de forma tímida, se desarrollan en el marco de una tendencia al tratamiento convencional de la información no sólo en el plano formal, sino también en la selección temática. De hecho, en la investigación se constata que "Sociedade" se constituye como el ámbito noticioso con mayor número de noticias incorporadas a portada -el 27 por ciento-, seguida de "Sucesos" -el 19 por cien-, "Política” -el 18

\footnotetext{
${ }^{3}$ Una de las mayores lacras del periodismo en los últimos años es la falta de credibilidad de los medios de comunicación. En este sentido, uno de los casos paradigmáticos, y que en cierto modo sustentan esa desconfianza hacia la prensa, es el de Jayson Blair. El 11 de mayo de 2003 el todopoderoso New York Times se vio obligado a publicar en primera plana una nota en la que se informaba que uno de sus periodistas asignados al área de noticias nacionales, el susodicho Jayson Blair, había inventados informaciones y entrevistas, además de cometer multitud de plagios en más de 70 noticias publicadas en el periódico desde su entrada en 1998. La opinión pública reaccionó con estupor al conocer que uno de los diarios más rigurosos e influyentes del planeta había permitido tales irregularidades.

${ }^{4}$ Los datos ofrecidos por la WAN en la cumbre de Filipinas -62 Congreso Mundial de Periódicos y 16 Foro Mundial de Editores-, en diciembre del año 2009, muestran una tendencia al descenso del consumo de diarios en los países desarrollados -especialmente en Europa y USA-, mientras crece en otros ámbitos. No obstante, los informes ofrecidos por la Asociación Mundial de Periódicos -conocida por WAN, sus siglas en inglésmuestran la necesidad de entender el diario como una actividad multimedia en expansión y en cambio.
} 
por cien- y "Economía” -el 13 por cien-. En cambio, en el análisis de contenido apenas se han encontrado en portada noticias relacionadas con tecnología, ciencia y comunicación. A continuación vemos, en números absolutos el resultado global del estudio de la temática de las noticias de portada.

Cuadro 1. Temas y frecuencia. Elaboración propia

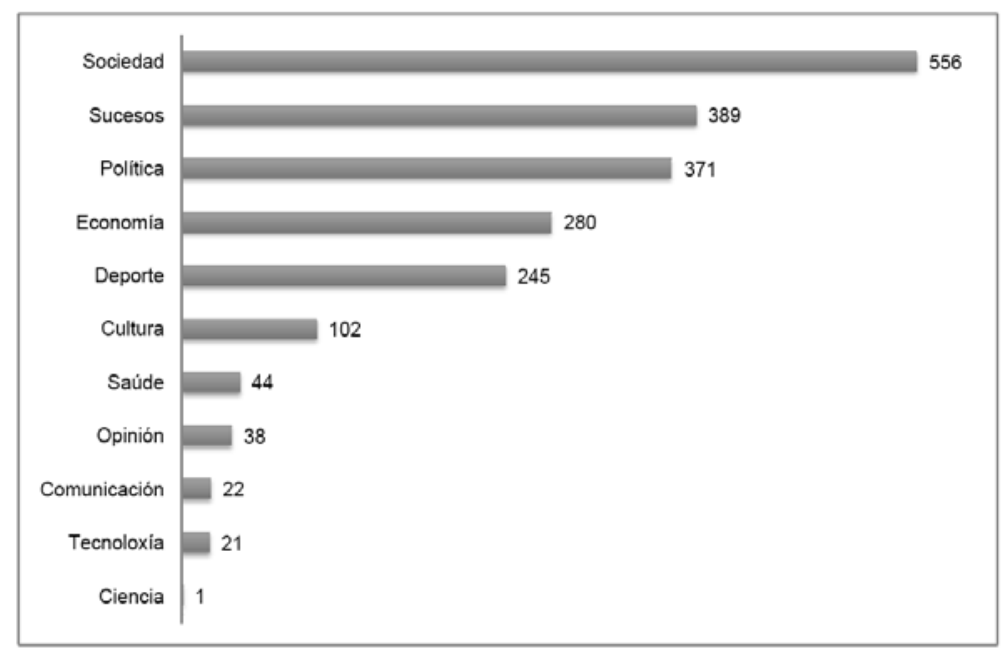

Este seguimiento de las pautas de los medios tradicionales -especialmente, de los diarios- también se refleja a la hora de aplicar las posibilidades que ofrece el hipertexto. De hecho, se detecta una incidencia reducida de la implantación de nodos y enlaces, aunque con tendencia al incremento progresivo.

\section{El llamado "Periodismo ciudadano" como experiencia para analizar}

Los responsables de los cibermedios gallegos no comparten las prácticas del denominado periodismo ciudadano, pero dicen seguir las experiencias que llevan adelante y aseguran que aprovechan aquello que estiman válido para hacer periodismo, especialmente algunas herramientas que se han popularizado en la llamada web social. De hecho, la convergencia tecnológica ha supuesto una verdadera revolución en la sociedad del siglo XXI, y los medios de comunicación también se han visto afectados tanto a nivel estructural como de contenidos.

En el momento actual, no solamente se han alterado los antiguos modelos de producción de noticias (Paterson y Domingo, 2008; Tremayne, Weiss y Alves, 2007), sino que también se han abierto de par en par las "puertas" del medio a contenidos generados por el usuario, permitiendo y fomentando la inclusión de comentarios, fotos, vídeos, blogs e incluso artículos elaborados por los lectores (para ejemplos e implicaciones de este hecho incontrovertible, véase DomINGo et al., 2008; HERMIDA y THURMAN, 2008; Thurman, 2008). Esta tendencia irrefrenable a aceptar las contribuciones de los lectores a la hora de construir el discurso noticioso de los medios puede ser denominado genéricamente como "Periodismo ciudadano" (Outing, 2005; DeuZe, 2008). 
Ya desde su nacimiento, el "periodismo ciudadano" ${ }_{5}$ ha captado de modo creciente la atención de los círculos académicos (CARPENTER, 2008; Deuze, Bruns y Neuberger, 2007; Domingo et al., 2008; Kovacic y ErJaVec, 2008). Los estudios, hasta el momento, se centran mayoritariamente en la naturaleza participatoria (DeUze et al., 2007; Domingo et al., 2008) y en el user-centered design (HERMIDA y Thurman, 2008) de este fenómeno, esbozado en la articulación de Rosen (2008): "When the people formerly known as the audience employ the press tolls they have in their possession to inform one another, that's citizen journalism”.

Aunque existe un cierto consenso a la hora de equiparar los términos Citizen journalism y Participatory journalism, hay quien, como Axel BRUNS, propone una distinción entre los términos "periodismo participativo" y "periodismo ciudadano". El periodismo participativo está más profundamente relacionado, de acuerdo con el autor, con el gatewatching - o selección de contenido para ciertas audiencias- y cómo Internet transformó progresivamente este proceso en una fiesta colectiva, formada completamente bajo la responsabilidad del periodista. Sin embargo, el periodismo ciudadano, para BRUNS:

"[...] aims to more accurately reflect a wider range of public views on specific issues through changes in the research and reporting approaches of journalists. The movement sees specially newspapers and their Websites as instrumental in developing a new form of 'civic commons' where solutions to existing problems are found through constructive debates that are orchestrated and led by editors and journalists on their pages” (BRuNs, 2005: 35).

Para Shayne Bowman y Chris Willis, autores del ensayo We Media: How audiences are shaping the future of news and information, el término "periodismo participativo" define el acto de un ciudadano, o un grupo de ciudadanos, con un rol activo en el proceso de recogida, análisis y difusión de noticias e información.

"Por periodismo cívico, comunitario, se entiende la acción de retomar contacto con la comunidad, descubriendo lo que los lectores quieren y abriendo espacios para charlas de temas de interés público. En el fondo subyace la idea de que la gente sea quien recabe la información y la transmita a otras personas" (Cit. en FeRnANDES, 2003).

La tendencia del "periodismo ciudadano" ha ganado exponencialmente espacio en los medios en los últimos años como una red social de trabajo gracias a las herramientas desarrolladas (GILLMOR, 2004); la evolución de las tecnologías ha propiciado la aparición de herramientas de comunicación que posibilitan que cualquiera pueda llegar a ser un periodista a bajo coste y con impacto universal. BOWMAN \& Willis (2003) proveen una cuidadosa definición del concepto que todavía trabaja con la forma en la que el periodismo es entendido hoy en día:

\footnotetext{
${ }^{5}$ El análisis del origen y evolución del denominado Periodismo ciudadano que se recoge en este artículo recoge los trabajos de documentación realizados por el investigador Moisés Limia, del grupo Novos Medios, en el marco de los proyectos de investigación que lleva adelante el grupo -en concreto, el subproyecto "Convergencia digital en los medios de comunicación. Medios”, del Ministerio de Educación y Ciencia (SEJ2006-14828-C06-03), y el ya citado "Tendencias na converxencia dos medios de comunicación en Galicia 20072010" (proyecto PGIDIT07PXIB212149PR, de la dirección general de I+D de la Xunta de Galicia)- y que han dado lugar a varias comunicaciones a congresos.
} 
"[Citizen journalism is] the act of a citizen, or group of citizens, playing an active role in the process of collecting, reporting, analyzing and disseminating news and information. The intent of this participation is to provide independent, reliable, accurate, wide-ranging and relevant information that a democracy requires. Participatory journalism is a bottom-up, emergent phenomenon in which there is little or no editorial oversight or formal journalistic workflow dictating the decisions of a staff" (BowMAn \& WiLLIS, 2003: 36).

Ya en 1995 Nicholas NEGROPONTE predijo en su libro Ser digital, que en el futuro las noticias en línea darían a los lectores la habilidad de escoger solo los temas y las fuentes que les interesan. Y parece que ese futuro ha llegado ya. Los lectores no solamente condicionan la agenda noticiosa, sino que incluso dirigen u orientan la práctica informativa. En el libro The Elements of Journalism, Bill KovAcH y Tim RosenstiEL explican que cada vez más la noticia es producida y condicionada por los lectores-usuarios. No en vano, el periodismo ciudadano es aquel que hace posible la participación activa de los actores sociales que intervienen en todo el procesamiento de la información de interés público. Por lo tanto, sus características esenciales son formar opinión pública mediante la creación de públicos deliberantes y promover la participación ciudadana.

El Periodismo participativo o ciudadano lleva implicada en su misma definición una tensión para el periodismo del siglo XXI: la lógica económica de construir plataformas participatorias de acceso libre para atraer grandes comunidades de usuarios, versus la lógica profesional de mantener la tradicional autoridad sobre la configuración del flujo informativo. Para Dan GILLMOR, periodista, bloguero y profeta del Periodismo 3.0, los modos tradicionales de producir información, gracias a los medios sociales y los instrumentos de publicación de ciberinformación, han sido trastocados para siempre: la audiencia detenta el poder.

"Las noticias son producidas por gente normal que tiene algo que decir y que mostrar. Ya no son sólo difundidas por los medios oficiales que han decidido tradicionalmente cómo será el primer esbozo de la historia. Ahora el primer borrador de la historia lo escribe la antigua audiencia” (GILLMOR, 2004).

Para los responsables de cibermedios gallegos y profesionales del ciberperiodismo que ofrecieron su punto de vista para esta investigación, el Periodismo participativo no es una moda pasajera y, por tanto, es una práctica que conviene seguir, aunque no imitar. Y, en especial, se muestran interesados por la utilización de las herramientas tecnológicas para la comunicación, edición, publicación e interacción cooperativa en Internet a fin de facilitar y promover la participación de los ciudadanos en la creación de contenidos en red.

\section{La red como espacio de deliberación pública}

La red es contemplada como espacio de deliberación pública, lo que lleva a los periodistas a seguir debates en la red y utilizar la red como fuente para algunas informaciones. En los últimos años la democratización de la producción online de los medios merece el escrutinio de científicos y académicos para entender sus efectos en el diálogo y en la participación en la esfera pública (HAAs, 2005), incluyendo los “públicos” localizados de comunidades rurales. El trabajo teórico y 
empírico, en los últimos años, ha demostrado que la deliberación pública puede promover una ciudadanía informada, puesto que posibilita que los ciudadanos se sientan atraídos por los temas, comparte la información y sopesa alternativas, todas ellas necesarias para construir una opinión pública formada que afecta al ejercicio de la política pública (FISHKIN, 1996; GASTIL, 2008).

Es de gran interés para nuestra concepción de la red como espacio democrático la definición conceptual arrojada por GASTIL (2008) de conversación pública deliberativa. El proceso social de deliberación extiende la dimensión de la mera expresión de personal de pensamientos e ideas hacia una discusión pública en la que un grupo de gente discute en conjunto sobre un tema. GaSTIL explica que en un proceso deliberativo, los individuos se ven a sí mismos como parte de una discusión pública, y ven a los otros como fuentes de información y reflexión. Así, existen 4 dimensiones clave para el éxito de un proceso social deliberativo:

1. El contexto debe asegurar una oportunidad adecuada para hablar a todos.

2. Los participantes tienen la obligación de considerar cuidadosamente las palabras que oyen.

3. La deliberación requiere una comprensión mutua en la que los participantes hablan con claridad y piden clarificaciones cuando son necesarias.

4. Un proceso deliberativo requiere participantes para mantener un grado de respeto consigo mismos y con sus compañeros de debate.

Una de las muchas vías posibles para promover la deliberación pública de asuntos de actualidad son los comentarios de los lectores. En particular, los comentarios pueden expresar testimonios de experiencias personales o especificar y clarificar asuntos individuales, necesarios en ocasiones para crear una solución efectiva (RYFE, 2005; GASTIL, 2008). Los lectores pueden también proveer una multiplicidad de perspectivas que resultaría difícil representar en un simple editorial. Esta diversidad de opiniones constituye un aspecto crítico de la deliberación pública (PRICE, CAPELLA \& NiR, 2002; MutZ, 2006) y de la solución de problemas públicos (PAGE, 2007). Finalmente, los comentarios de los lectores constituyen una herramienta interactiva entre los periodistas y el público. Tales conversaciones pueden proveer un importante punto de conexión, reconociendo de este modo el potencial de los ciudadanos para contribuir a la discusión de temas políticos más que reducirlos a la posición de audiencia pasiva. Sería una conversación entre expertos (CAMPBELL, 2000: 691).

Los comentarios, la creación de foros y chats, los weblogs... han democratizado la publicación online y han posibilitado que los lectores-usuarios puedan informar y opinar en un formato que derriba los antiguos muros que tradicionalmente separaban a periodista y audiencia. Así, el periodismo cívico es lo más parecido a una conversación entre iguales.

“Convertir la información en una conversación, de muchos para muchos en lugar del uno para muchos. Del broadcasting o comunicación de masas al we media o periodismo ciudadano: de muchos a muchos, es el lema” (VARELA, 2005: 32).

Hasta tal punto la información ha vuelto a sus propietarios originales -los 
lectores, el público-, que la gran conversación global, de todos a todos, que facilitan las herramientas tecnológicas de la llamada web semántica permite que los propios lectores-usuarios se erijan en perros guardianes que detentan una función de control y tutela moral sobre los medios de comunicación. Solamente así es posible entender que la blogosfera haya conseguido terminar con la carrera profesional de periodistas tan prestigiosos como Dan RATHER ${ }^{6}$ o Eason JORDAN; o cómo ha podido desenmascarar a Jeff GANNON, en realidad Jeff GUCKHERT ${ }^{8}$. Esto ha sido posible debido a la progresiva incorporación de fórmulas participativas en los medios tradicionales. Ya en 2005, el New York Times demostró tener un más que vivo interés en la blogosfera cuando en 2005 adquirió por 400 millones de dólares el portal About.com, que contenía más de 500 blogs sobre diversos temas y que recibía 22 millones de visitas al mes.

\section{La interactividad y la participación como motores del periodismo}

Las características definitorias de los cibermedios son la inmediatez, la hipertextalidad, su perfil multimedia y la interactividad (Díaz NocI, 2001:25). Aun reconociendo la importancia de todas y cada una de ellas, resulta obvio que la interactividad es el concepto clave de la era digital (NEGROPONTE, 1995) y del periodismo ciudadano. La interactividad significa un cambio de la comunicación uno-a-muchos de los medios tradicionales hacia el flujo comunicativo que emerge con el modelo de comunicación de dos "vías" que convierte a las audiencias en consumidores activos (PAVLIK, 2001). El potencial de la interactividad para facilitar un diálogo entre los medios y sus audiencias es, por tanto, indisputable.

En la literatura científica existe una extensa discusión acerca de las definiciones de interactividad (Heeter, 1989; Massey y Levy, 1999; McMillan, 2006; Rafaeli y Sudweeks, 1997; Steuer, 1992). Parece imponerse la visión de la interactividad como un constructo multidimensional (LEE, 2000; MASSEY y LeVY, 1999; McMillan, 2006; Schultz, 2000; Stromer-Galley, 2000). En particular, nos parece muy interesante la división que McMillan aporta acerca de las tres dimensiones de la interactividad: user-to-user, user-to-document y user-to-system.

${ }^{6}$ Dan Rather, célebre presentador de informativos de la CBS, líder de audiencia durante más de 25 años y uno de los periodistas más influyentes de EEUU, se vio forzado a dimitir en 2005 cuando durante la campaña presidencial mostró en su informativo unas cartas que supuestamente demostraban un trato de favor con George Bush cuando éste realizaba el servicio militar. Un blogger demostró que la tipografía empleada en las cartas todavía no se había creado en el año en que estaban fechadas. El 10 de marzo de 2005 Rather pidió disculpas públicamente en su informativo, que sería el último.

${ }^{7} \mathrm{Al}$ igual que en el caso de Dan RATHER, otro blog -Captain's Quarter- forzó la dimisión del periodista Eason Jordan, al recoger unas declaraciones en el Foro Económico Mundial de Davos en las que el jefe de informativos de la CNN afirmaba que los soldados norteamericanos habían matado deliberadamente a doce periodistas en Irak.

${ }^{8}$ Los bloggers desenmascararon a Jeff Gannon, un supuesto periodista que estaba siempre acreditado en las ruedas de prensa de la Casa Blanca y que lanzaba estratégicamente preguntas “amables” al presidente de EEUU para que saliese con éxito de situaciones comprometidas. 
Los académicos han investigado con profusión sobre el potencial del periodismo online para emplear herramientas interactivas en una senda que puede promover -y promueve, de hecho- la participación ciudadana (SCHULTZ, 1999; ROSEBERRY, 2005; NIP, 2006). Estos estudios identifican las herramientas interactivas de acuerdo a tres dimensiones del periodismo público: “conectar con la comunidad, atrayendo/ interesando a los individuos como ciudadanos y ayudando la deliberación pública en la búsqueda de soluciones” (NIP, 2006).

Como hemos indicado en páginas precedentes, el modelo pasivo y unidireccional empleado tradicionalmente por los medios de comunicación encuentra actualmente algún rechazo y busca la superación mediante el concepto de usuario activo, que busca contenidos, navega y aporta información. Así, los principales medios han adoptado mayoritariamente prácticas de periodismo participativo (HeRmida y Thurman, 2008; Thurman, 2008). De este modo, es posible aprovechar las potencialidades de interacción que ofrece el nuevo medio digital. Además, las tecnologías interactivas han abierto nuevas vías para incorporar el contenido generado por los usuarios dentro del contexto de las noticias.

Aquí emerge con fuerza el contenido generado por los usuarios, que en ocasiones llega a determinar la orientación de un medio ante un determinado acontecimiento o hecho noticioso. Aunque la investigación de cariz académico sobre UGC (User Generated Content) está todavía en una etapa temprana, iniciática, algunos estudios pioneros han mostrado que los medios de noticias online consideran el UGC como un componente importante del periodismo digital (CHUng, 2007; Domingo, 2008; HERMidA y THURMAN, 2008; ORNEBRING, 2008; THURMAN, 2008).

El estudio sobre periodismo online y medios interactivos se ha centrado en los blogs (Barlow, 2008; Bloom, 2003; Kline, Burstein, De KeiJzer \& Berger, 2005; ReEse, Rutigliano, Hyun \& JeOng, 2007). La investigación académica se he centrado menos, sin embargo, en el contenido de los comentarios de los lectores en los sites de los periódicos. Ésta parece ser una vía de estudio diáfana para los próximos años, pues la sección de comentarios de los lectores en la opinión periodística online tiene un potencial enorme para encarnar un espacio de deliberación pública, ofreciendo a los ciudadanos nuevas vías de participación en la esfera pública dentro del contexto del periodismo como servicio público.

La importancia de los comentarios radica en el hecho de que la posibilidad de publicar comentarios en los sites noticiosos no solamente facilita una comunicación entre periodistas y lectores-usuarios (user-to-user interactivity) sino que también permite a los usuarios crear su propio contenido (user-to-document interactivity). Para cada una de estas dimensiones, McMillan (2006) ofrece un modelo organizado en cuatro partes que refleja el nivel de control de los receptores yuxtaponiendo la dirección de la comunicación (one-way vs. Two-way) en el caso de la interactividad user-to-user y la naturaleza de la audiencia (activa vs. Pasiva) en el caso de interactividad user-to-document.

\section{Algunos rasgos del nuevo escenario}

Las emergentes tecnologías de la comunicación han alterado el paisaje de la 
producción de la información y distribución de modo significante (SHIRKY, 2008), tensando y a veces desplazando el rol tradicional del periodismo de informar al público (véase, por ejemplo, TuMBER). De hecho, el advenimiento del denominado Periodismo ciudadano ha abierto la vía para que los ciudadanos participen aportando informaciones y noticias (especialmente en los medios llamados hiperlocales) y para que reflejen todo tipo de acontecimientos con sus teléfonos móviles. Esto es, se supera la tradicional actitud pasiva del lector que recibía acríticamente la versión aportada por los diferentes medios de comunicación. Incluso pueden escribir sus propios blogs en el marco del periódico, contribuyendo de ese modo a la creación de contenidos originales (crowdsourcing).

Algunas de estas herramientas que permiten la participación también las han incorporado los cibermedios gallegos, que muestran especial interés por la gestión de los blogs -considerados la avanzadilla de la web 2.0-, los foros en línea -ámbitos de opinión en línea-, las iniciativas de micro-blogging -servicio que permite a los usuarios enviar mensajes breves-, las redes sociales -estructuras sociales virtulaes que se establecen en internet-, y los sistemas de agregación de contenidos -un tipo de software que permite suscribirse a fuentes de noticias en formatos RSS, Atom y otros derivados de XML/RDF-.

Los cibermedios gallegos avanzan lentamente en este terreno, pero muestran una tendencia ascendente en los análisis realizados en el año 2009. Los datos apuntan que más de un tercio de los cibermedios estudiados incorporan las redes sociales en sus estrategias y los comenarios de noticias se aproximan a este porcentaje.

Cuadro 2. Elementos interactivos. Elaboración propia

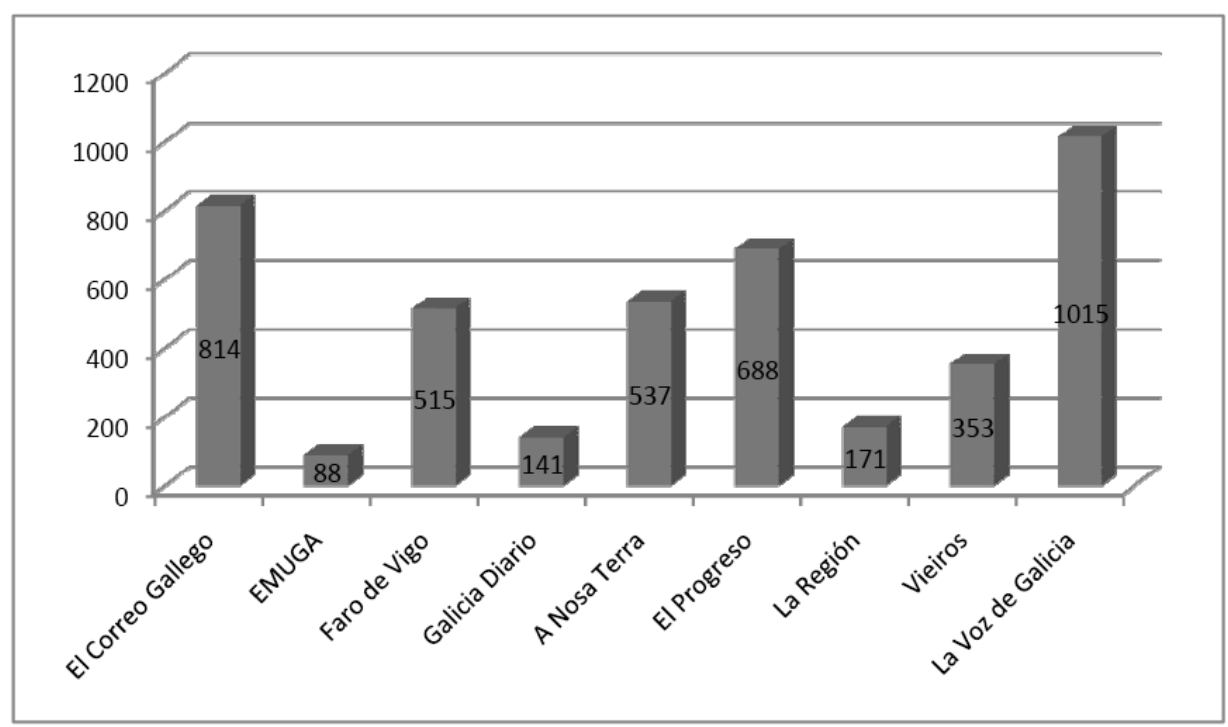

A pesar de las limitaciones en la incorporación de nuevas herramientas y elementos interativos, no cabe duda que los cibermedios ya contemplan en su estrategia esta vía y que parecen decididos a dar nuevos pasos en esta dirección. Los 
responsables de los cibermedios gallegos consideran necesaria la experimentación y la búsqueda de nuevas formas de ofrecer la información para incrementar el número de usuarios y, por tanto, para alcanzar una mejor aceptación de los productos que elaboran.

\section{Conclusión}

En el momento actual, el periodismo vive una metamorfosis que le abre nuevos espacios y lo coloca ante nuevos desafíos. La fase de cambios y transformaciones que caracteriza el ámbito de la comunicación mediada tecnológicamente en el siglo XXI ha modificado sustancialmente el escenario periodístico no sólo en lo que a movimientos y corrientes se refiere, sino a los propios debates sobre el rol que debe cumplir el periodismo en la compleja sociedad actual, que presenta diferencias importantes con la del pasado siglo. Lo que permanece es lo básico, la esencia del periodismo - "el periodismo puro y duro"-, y la necesidad de los ciudadanos de disponer de información para poder moverse en la sociedad.

El periodismo pertenece a la sociedad y por tanto evoluciona con la sociedad sin perder la esencia, en viejos y nuevos soportes, con viejas y renovadas técnicas, y con viejos y nuevos desafíos. El periodismo, siempre cambiante, se reinventa cada vez que precisa dar respuestas y ofrecer alternativas. Y lo hace con el peso de una herencia cosechada en escenarios muy diversos. El objetivo es ofrecer información de interés público, explicarla, interpretarla y valorarla. Y hoy, al igual que en el pasado, conviven numerosas tendencias y movimientos periodísticos, que en muchos casos buscan otro periodismo posible.

Lo que se ha denominado "Periodismo ciudadano", al margen de si denominación es más o menos afortunada, no cabe duda que ha permitido la aparición de nuevos proyectos informativos y ha fomentado el empleo de herramientas que pueden conducir a un periodismo más participativo, lo que incide en las estrategias de los cibermedios que apuestan por el modelo convencional -el que considera central la intervención del profesional del periodismo para elaborar la información-. Aunque el periodismo es básicamente mediación profesional y mantiene claras diferencias con la publicidad y la propaganda, las nuevas herramientas también pueden emplearse para elaborar información de calidad. Y este es un aspecto que forma parte de las esencias del periodismo: la veracidad y la calidad de todos los elementos -textuales y formales- que integran el mensaje informativo.

El debate, sin duda, está abierto y estimula interesantes propuestas y proyectos periodísticos que buscan mostrar la validez de sus planteamientos. Y en la actualidad un número importante de cibermedios incorpora herramientas de la web social, como se constata en la investigación sobre los cibermedios gallegos. Ciertamente, cada proyecto periodístico emplea estas herramientas con finalidades diferentes y en el marco de iniciativas muy dispares. Pero en todos los casos estas herramientas resultan útiles para el ejercicio responsable de nuestra profesión.

Aunque los cibermedios gallegos ofrecen información diseñada desde pautas bastante convencionales en el campo formal y en el campo temático, hay aspectos 
innovadores tanto en la aparición de elementos interactivos como en la incorporación de las nuevas herramientas que permiten la elaboración de un periodismo de calidad elaborado por profesionales, pero con ventanas abiertas a la participación de los ciudadanos. La experimentación para el cambio periodístico en los cibermedios gallegos se puede decir que avanza, aunque sin muchas prisas y en el marco de una estrategia que no quiere asumir muchos riesgos. Es, en todo caso, una opción.

\section{Referencias bibliográficas}

BOWMAN, S. \& WILLIS, C. (2003): "We Media: How audiences are shaping the future of news and information”, en Hypergene: http://www.hypergene.net/ wemedia/download/we_media.pdf. [fecha de consulta: 1 de diciembre de 2009].

BRUNS, A. (2005): Gatewatching: Collaborative online news production. NewYork, Peter Lang.

CAMPBELL, C. C. (2000): "Citizens Matter: and that is why public journalism matters", en Journalism Studies, 1 (4). pp. 689-694.

CARPENTER, S. (2008): "How online citizen journalism publications and online newspapers utilize the objectivity standard are rely on external sources”, en Journalism \& Mass Communication Quaterly, 85 (3). pp. 531-548.

DEUZE, M.; BRUNS, A. \& NEUBERGER, C. (2007): "Preparing for an age of participatory news", en Journalism Practice, 1 (3), pp. 322-338.

DEUZE, M. (2008): "The professional identity of journalists in the context of convergence culture”, en Observatorio, 2 (4). pp. 103-117.

DÍAZ NOCI, J. (2001): La escritura digital. Hipertexto y construcción del discurso informativo en el periodismo electrónico. Bilbao, Universidad del País Vasco.

DÍAZ NOCI, J. \& PALACIOS, M. (2008): Metodologia para o estudo dos cibermeios. Salvador de Bahia. Brasil, EDUFBA - Editora da Universidade Federal da Bahia-.

DOMINGO, D.; QUANDT, T.; HEINONEN, A.; PAULUSSEN, S.; SINGER, J. B. \& VUJNOVIC, M. (2008): "Participatory journalism practices in the media and beyond”, en Journalism Practice, 2 (3). pp. 326-342.

FERNANDES, M. (2003): "Periodismo cívico: pasado, presente y futuro", en Chasqui $\mathrm{n}^{\circ}$ 82: http://chasqui.comunica.org/content/view/172/63/. [fecha de consulta: 28 de noviembre de 2009].

GASTIL, J. (2008). Political Communication and Deliberation. Sage.

HAAS, T. (2005): “From 'public journalism' to the 'public's journalism'? Rhetoric and reality in the discourse on weblogs", en Journalism Studies, 6 (3). pp. 387396.

HERMIDA, A. \& THURMAN, N. (2008): "A clash of cultures: The integration of user-generated content in the discourse on weblogs", en Journalism Studies, 6 (3). pp. 387-396.

KOVACH, B. \& ROSENSTIEL, T. (2003): Los elementos del periodismo. Madrid, Ed. El País. 
KOVACIC, M. P. \& ERJAVEC, K. (2008). "Mobi journalism in Slovenia”, en Journalism Studies, 9 (6). pp. 874-890.

MUTZ, D. C. (2006). Hearing the other side: Deliberative vs. Participatory Democracy. New York, Cambridge University Press.

NEGROPONTE, N. (1995): El mundo digital. Barcelona, Ediciones B.

OUTING, S. (2005): “The 11 layers of citizen journalism”, en Poynter online: http://www.poynter.org/content/content_view.asp?id=83126. [fecha de consulta: 3 de diciembre de 2009]

PAGE, S. (2007). The Difference: How The Power of Diversity Creates Better Groups,Teams, Schools, and Societies. Woodstock, Princeton University Press.

PATERSON, C \& DOMINGO, D. (2008): Making online news: The etnography of new media production. New York, Peter Lang.

PRICE, V., CAPPELLA, J. N., \& NIR, L. (2002): "Does disagreement contribute to more deliberative opinion?”, en Political Communication, 19 (1). p. 95.

RYFE, D. (2005). “Does deliberative democracy work?”, en Annual Review of Political Science, 8. pp.49-71.

TREMAYNE, M.; WEISS, A. S. \& ALVES, R. C. (2007): "From product to service: The diffusion of dynamic content in online newspapers", en Journalism \& Mass Communication Quaterly, 84 (4). pp. 825-839.

THURMAN, N. (2008): "Forums for citizen journalists? Adoption of user generated content initiatives by online news media”, en New Media \& Society, 10 (1). pp. 139-157. 